\title{
Blast effects and protective technology design
}

\author{
M. Zineddin \\ Institute for International and Civil Security, and \\ Department of Civil Infrastructure and Environmental Engineering, \\ Khalifa University of Science, Technology and Applied Research, \\ Abu Dhabi, UAE
}

\begin{abstract}
The fundamental goal of protective construction is to improve the probability of survival of people and other contents in a given facility for a given threat. It is important to realize that the protective building is the last layer of defense against a threat and that all other protective measures (intelligence, law enforcement, surveillance, barriers, etc.) have failed if the threat can be projected onto a facility. This implies that a designer must "know" the threat before conceptualizing the design and this may not be possible in many cases. Attackers can use various weapon systems in different combinations and such events cannot be predicted. However, using reliable information and objective threat and risk assessment can produce effective estimates of such incidents.

Usually, a facility design is based on a standard threat (for example, a specific bomb at a given stand-off distance). In other cases, a statistical approach, requiring that a specific percentage of facilities and contents will survive if a site is attacked, may be employed.

Physical security can be achieved by a variety of means and devices with a wide range of capabilities. These capabilities can be used to enable detection, deterrence, delay, and prevention of hostile activities. Structural hardening is a passive defense capability; it is only one aspect of these considerations and should be addressed in the broader context of physical security. As with any other fortification technology, passive defense alone cannot be used to protect against mobile and constantly varying threats.

A structure must be designed to prevent catastrophic failure and to protect its contents (personnel and equipment) from the effects of an explosion. Such effects may include nuclear and thermal radiation, electromagnetic pulse (EMP), air blast, ground shock, debris, fragments, and dust (protection from chemical
\end{abstract}


and biological (CB) threats should be considered, as appropriate). In order for a military facility to survive, the continuation of its operational mission must be ensured. For civilian facilities, however, the main concern is protecting people and/or critical assets. Therefore, survivability requirements (criteria) vary from one type of facility to another.

Keywords: blast effects, protective design, risk mitigation, physical security, antiterrorism protection, hardened structure.

\section{Introduction}

It is neither realistic nor cost effective to try to prevent or protect against every negative event that may occur within or against a target. There are four key components to full spectrum risk mitigation as illustrated in Figure 1.

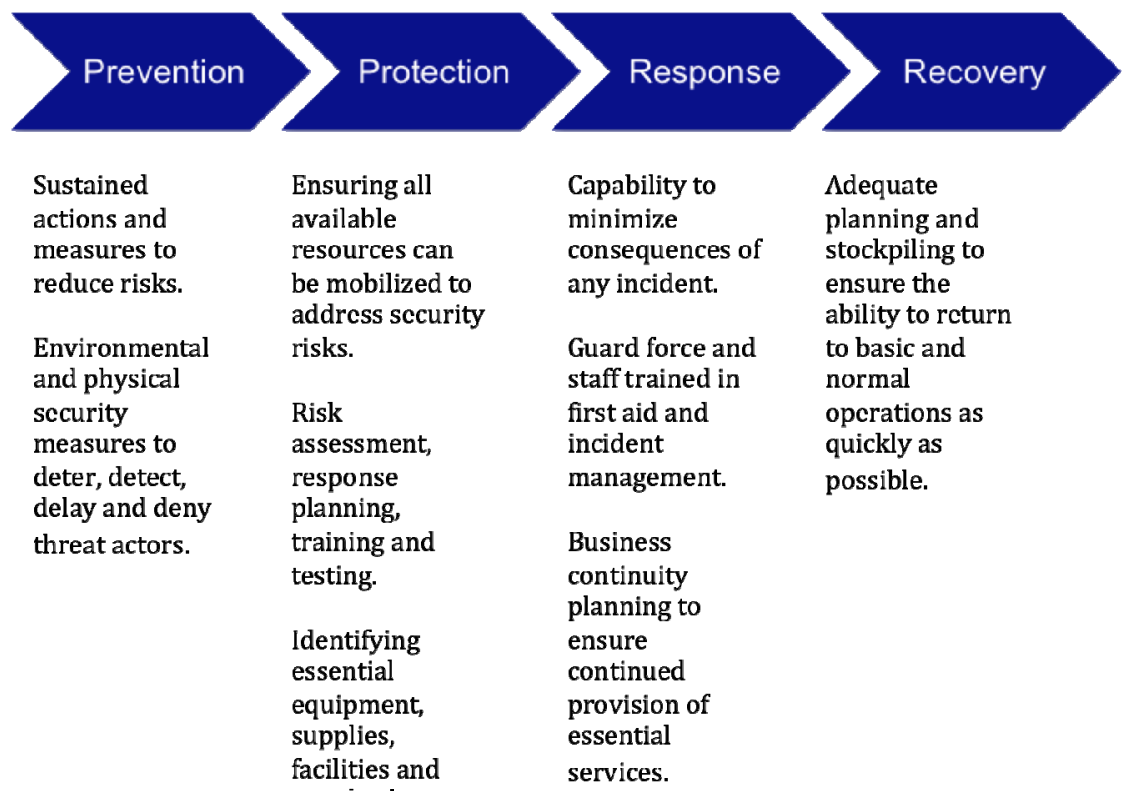

Figure 1: $\quad$ Full spectrum of risk mitigation.

As these examples indicate, full spectrum risk mitigation works across a number of different areas and disciplines, providing a "layered" approach to security within the compound. To maximize the effectiveness of this approach, security measures should be considered and embedded where possible in the following areas:

Urban planning: Harmonization with the security recommendations in building codes, standards and guidelines, and international best practice for similar sites. 
Building design: Protective design of structures, including adequate standoff, designing against progress collapse and providing adequate emergency egress routes.

Policies and procedures: Compliance with regulatory regimes and coordination with relevant government agencies, as well as the development of crisis management and business continuity planning.

Technology applications: CCTV analytics, smart access control systems, automatic number plate recognition systems, passive screening, emergency alerting and communications systems.

People: Educational awareness campaigns to report abandoned bags, suspicious behavior, adequate training and vetting of staff and security guards.

\section{Explosion effects}

An explosion is an extremely rapid release of energy in the form of light, heat, sound, and a shock wave. Explosive pressures encountered in design are typically much greater than other loads that are considered, but they decay extremely rapidly with time and space. As a rule of thumb, the pressures generated by the shock wave increase linearly with the size of the weapon, usually measured in equivalent pounds of TNT, and decrease exponentially with the distance from the explosion. The security duration of the explosion is extremely short, measured in thousandths of a second, or milliseconds.

As the shock wave expands, the incident or overpressure decreases. When it encounters a surface that is in line-of-sight of the explosion, the wave is reflected, resulting in a tremendous amplification of pressure on the surface of the object: shock waves can reflect with an amplification factor of up to about 12. The magnitude of the reflection factor is a function of the proximity of the explosion and the angle of incidence of the shock wave on the surface (with incidence normal to the targets resulting in the maximum pressure). Late in the explosive event, the shock wave becomes negative, followed by a partial vacuum, which creates suction behind the shock wave that can cause windows to fall outwards. For a specific type and weight of explosive material, the intensity of blast loading will depend on the distance and orientation of the blast wave relative to the protected space. These characteristics are aspects of the site size and placement of the building(s). Figure 2 illustrates the various blast loads on a building [1].

Immediately following the vacuum, air rushes in, creating a powerful wind or drag pressure on all surfaces of the building. This wind picks up and carries flying debris in the vicinity of the detonation. In an external explosion, a portion of the energy is also imparted to the ground, creating a crater and generating a ground shock wave analogous to a high-intensity short-duration earthquake. 


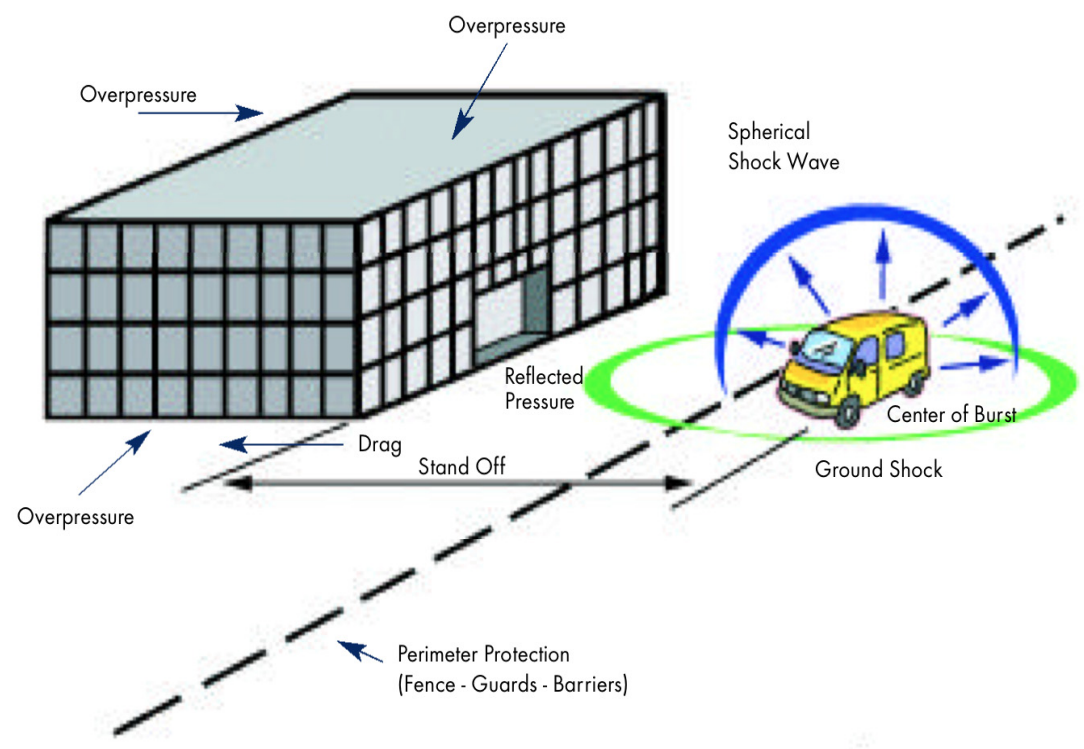

Figure 2: $\quad$ Blast loads on a building.

Since the indications are that explosive devices will continue to be a primary hazard, the emphasis in this will be on blast, shock, and impact. Structural design for safety and physical security requires a sound background in fortification science and technology. One must realize that loading environments associated with many relevant threats (impact, explosion, penetration, etc.) are extremely energetic, and their duration is measured in milliseconds (i.e., about one thousand times shorter than typical earthquakes). Structural response under short-duration dynamic effects could be significantly different from the much slower loading cases, requiring the designer to provide suitable structural details. Therefore, one must explicitly address the effects related to such sever loading environments, in addition to considering the general principles used for structural design to resist conventional loads. One must be familiar with the background material on structural consideration and design, and the experience gained from recent terrorist bombing incidents. Figure 3 illustrates the parameters of a typical blast wave [1].

\section{Security design}

Physical security consists of measures taken to address criminal and vandal threats. Physical Security uses defensive measures that provide layers of detection and delay around an asset. The defensive layer must provide enough delay time to allow a response force to halt the attack. Physical Security is 


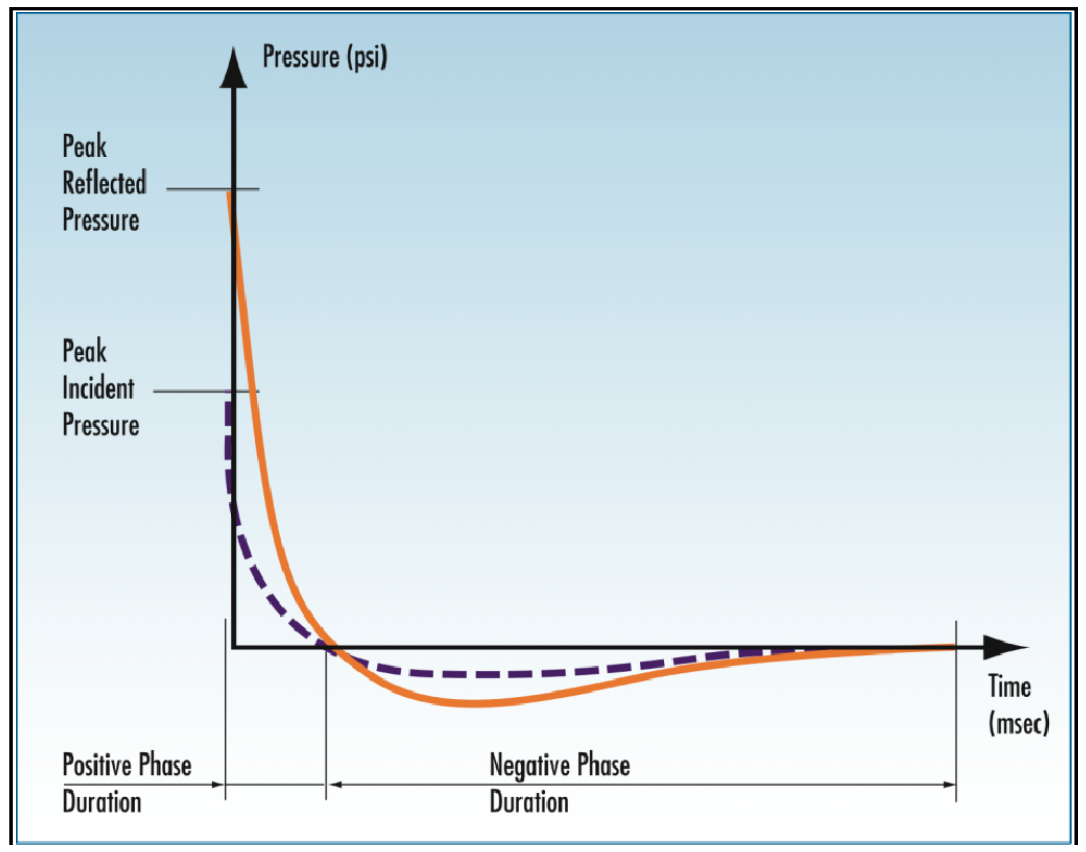

Figure 3: $\quad$ Blast wave parameters.

addressed primarily by policy that defines operational procedures, electronic security systems, and structural security measures to provide the required delay time. The assumption is that some minimal level of protection is required and risk is evaluated on an organization-wide basis with the assumption that there is always a criminal threat.

Antiterrorism protection addresses the design of both the building and the site to minimize the blast loads and weapon effects from terrorist threats to assets - usually people. This may mean the building is destroyed, but damage to assets is minimized. The actual threat to a specific asset is seldom known and it is unlikely that a specific asset will ever have a terrorist attack. The price people are willing to pay for protection from an unlikely threat of unknown magnitude has historically been very little in the world, but it is changing. As part of Antiterrorism Protection, blast hardening is sometimes done, but does not commonly meet the level of protection in the following definition of a hardened structure.

A hardened structure is usually designed to perform its primary mission after a wartime attack making hardening one of its primary requirements and a significant part of its cost. The facility is protected against a wide range of threats including forced entry, Chemical/ Biological/Radiological (CBR), airblast, ground shock, penetration, fragmentation, and damage to the structure and equipment due to explosive loading. Designs must consider how camouflage, concealment and deception, active defense, and manned response can reduce or limit the effectiveness of the threat. The design assumptions are 
that during a war, the facility will be attacked and that it must survive and function after the attack. Almost all hardened structures inherently satisfy the requirements for both Physical Security and Antiterrorism Protection.

\section{Protective design}

Generally, the following issues should be addressed to protect valuable assets. The first is maximizing standoff distance to reduce the blast and fragmentation loads on the structure under consideration. If sufficient space is available, this may be the most effective mitigation approach, but it requires one to ensure that the protecting perimeter is secured to ensure the specified distance. The next most important issue to consider is the prevention of building collapse, and this requires careful attention to the structural layout and design details. Once these items are addressed, one must minimize hazardous flying debris such as glass, dislodged structural parts, and nonstructural components such as furniture and equipment. Providing an effective building layout also can contribute significantly to protecting valuable assets. This may be achieved by placing less valuable assets closer to hazards and more valuable assets farther from hazards. Providing protected spaces to enable people to take quick refuge until further instructed from security and/or rescue personnel is known to be very effective. Limiting airborne contamination should be considered, as appropriate. Additionally, one should address requirements for fire hazard mitigation and effective evacuation, rescue, and recovery operations. Providing mass notification is essential to prevent panic and assist in post-incident activities. Finally, one should consider options to facilitate upgrades, as might be required by periodic risk assessments [2].

A protected facility consists of several components: a protected perimeter, a protective structures, essential subsystems, and nonessential support subsystems. After all non-structural considerations are addressed; another specific issue that must be considered to protect a facility is blast- and shock-resistant design of the structure to protect contents from the effects of blast, shock, radiation, fragments, debris, dust, etc. It should be clear that the survival of the structure alone may not be sufficient if damage to the contents or personnel exceeds the survivability criteria. In order to consider all these factors, a designer should know very accurately what is expected of a facility.

The term hardness and survivability define the capability of a protective system (or facility) to resist the anticipated effects and meet the protection criteria. Survivability can be increased by enhancing a facility's hardness or other protective features. Other means for achieving the same goal can be employed as well (for example, redundancy, ensuring a larger standoff distance, location of other sites, etc.). Placing another protective layer between the facility or its contents and the weapon also can be very helpful because that layer will absorb some of the undesirable effects. Burying a facility in rock or soil will provide major benefits by reducing weapon effects on the system and/or by making it harder to locate the target [3]. 
A designer should employ protection criteria to ensure that the facility and its contents will not be subjected to environments (motions, stresses, etc.) beyond a certain limit.

\section{Design strategies}

The design process for protective facilities is described as follows:

1. Define facility operational performance requirements.

2. Establish quality assurance (QA) criteria for analysis, design, and construction work, and assign responsibilities for various activities throughout the entire project.

3. Perform threat, hazard, and risk assessment and determine future risk assessment reviews.

4. Determine explosive sources and their locations and magnitudes.

5. Estimate corresponding loading conditions.

6. Establish general siting, facility layout, and design criteria.

7. Proportion members for equivalent static loads.

8. Compute blast loads on facility more accurately.

9. Compute loading from fragments.

10. Compute loading from crater ejecta.

11. Compute loading from ground shock.

12. Combine all dynamic loads and perform preliminary dynamic analyses.

13. Redesign facility to meet protective criteria under these dynamic effects.

14. Consider nuclear radiation, EMP, thermal effects, CB, etc., if appropriate.

15. Verify design by acceptable methods.

16. Prepare design documentation for shop and fieldwork.

17. Embark on contracting and construction activities; activate appropriate QA.

18. End-of-construction inspection and review.

19. Facility begins its service life.

The design of a facility usually requires interaction among specialists in several disciplines, such as security, architectural, structural, mechanical, electrical, electronics, and hardening. This effort utilizes a team approach to ensure that all possible aspects of the problem have been considered and that the proposed combined plan optimizes the available solutions in the various areas.

A rational approach for selecting appropriate protective measures for an asset is based on comparing the cost of the mitigation with the cost of the consequence if no improvements are made in protecting the asset. Initially, small investments in protecting an asset can provide significant benefits. However, additional protection enhancements will become increasingly more expensive. 


\section{References}

[1] Lane, R., Craig, B. \& Babcock, W., Materials for Blast and Penetration Resistance. Material Ease, the AMPTIAC Quarterly, Volume 6, Number pp. 39-45, Rome, NY, 2001.

[2] Krauthammer, T., et al, Structural Design for Physical Security: State of the Practice, Structural Engineering Institute, American Society of Civil Engineers: Reston, Virginia, pp. 3-7 to 3-13, 1999.

[3] Federal Emergency Management Agency, FEMA 426, Building Design Guidance (Chapter 3). Washington, D.C, 2003. 